\title{
Mycovirus-Induced Hypervirulence of Leptosphaeria biglobosa Enhances Systemic Acquired Resistance to Leptosphaeria maculans in Brassica napus
}

\author{
Unnati A. Shah, ${ }^{1}$ loly Kotta-Loizou, ${ }^{1,2,+}$ Bruce D. L. Fitt, ${ }^{1}$ and Robert H. A. Coutts ${ }^{1}$ \\ ${ }^{1}$ Department of Biological and Environmental Sciences, School of Life and Medical Sciences, University of Hertfordshire, \\ Hatfield, Hertfordshire, AL10 9AB, U.K. \\ ${ }^{2}$ Department of Life Sciences, Faculty of Natural Sciences, Imperial College London, London SW7 2AZ, U.K. \\ Accepted 21 October 2019.
}

\begin{abstract}
Phoma stem canker (blackleg) is one of the most important diseases of winter oilseed rape (Brassica napus) worldwide and is caused by a complex that comprises at least two species: Leptosphaeria maculans and L. biglobosa. Screening a panel of field Leptosphaeria isolates from $B$. napus for the presence of mycoviruses revealed the presence of a novel double-stranded RNA quadrivirus in L. biglobosa and no viruses in $L$. maculans. Following elimination of the mycovirus, virus-infected and virus-free isogenic lines of $L$. biglobosa were created. A direct comparison of the growth and virulence of these isogenic lines illustrated that virus infection caused hypervirulence and resulted in induced systemic resistance toward $L$. maculans in $B$. napus following lower leaf preinoculation with the virusinfected isolate. Analysis of the plant transcriptome suggests that the presence of the virus leads to subtle alterations in metabolism and plant defenses. For instance, transcripts involved in carbohydrate and amino acid metabolism are enriched in plants treated with the virus-infected isolate, while pathogenesis-related proteins, chitinases and WRKY transcription factors are differentially expressed. These results illustrate the potential for deliberate inoculation of plants with hypervirulent $L$. biglobosa to decrease the severity of Phoma stem canker later in the growing season.
\end{abstract}

Keywords: fungus-plant interactions, systemic acquired resistance

In natural situations, plants are continually exposed to and can become infected with pathogens, and such infections can have consequences for subsequent infections with either closely related or unrelated pathogens. In some cases, the roots of crop plants are protected from diseases caused by soilborne

${ }^{\dagger}$ Corresponding author: I. Kotta-Loizou; i.kotta-loizou13@imperial.ac.uk

Funding: Additional financial support for B. Fitt was provided by the Biotechnology and Biological Sciences Research Council ERA-CAPS, grants BB/N005112/1, BB/M028348/1, BBP00489X/1, and BB/I017585/2.

*The $\boldsymbol{e}$-Xtra logo stands for "electronic extra" and indicates that seven supplementary figures and two supplementary tables are published online.

The author(s) declare no conflict of interest.

Copyright $\odot 2020$ The Author(s). This is an open access article distributed under the CC BY 4.0 International license. beneficial microorganisms, including fungi, and demonstrate induced systemic resistance, which differs from systemic acquired resistance (SAR), caused by a hypersensitive response triggered by plant pathogens (Pieterse et al. 2014). Thus, it is reasonable to assume that interactions between closely related fungal species that elicit necrosis can result in SAR and that this is a naturally occurring phenomenon. Additionally, the level of SAR induced by the fungus infecting the plant initially might be increased due to the presence of mycoviruses that can cause hypervirulence. Indeed, the induction of hypervirulence caused by mycovirus infection of fungi, while thought to be a rarity, is now considered to be much more prevalent than originally suspected, and several examples have been described recently (Kotta-Loizou and Coutts 2017a; Okada et al. 2018).

Phoma stem canker (blackleg) is an internationally important disease of oilseed rape (Brassica napus; canola or rapeseed), causing serious losses in Europe, Australia, and North America. For instance, United Kingdom losses of approximately $£ 100$ million per season have been estimated using national disease survey data and a yield loss formula (Fitt et al. 2006a). Phoma stem canker pathogen populations comprise two main species: Leptosphaeria maculans, associated with damaging stem base cankers, and L. biglobosa, often associated with less-damaging upper stem lesions (Fitt et al. 2006a). Disease control is achieved by growing cultivars with quantitative or qualitative resistance, the latter of which is controlled by resistance genes (e.g., Rlm genes against L. maculans). L. maculans and $L$. biglobosa not only coexist in oilseed rape (Fitt et al. 2006b; West et al. 2002) but also potentially coinfect the same site on plants (Mahuku et al. 1996); therefore, it is reasonable to propose that induction of host resistance against $L$. maculans also occurs naturally following simultaneous infection with L. biglobosa. It is known that pathogens closely related to challenge isolates are better inducers of resistance than those which are nonpathogens or pathogens of unrelated hosts (Mahuku et al. 1996). For instance L. biglobosa, which is not economically destructive, appeared to be effective in inducing resistance to $L$. maculans in oilseed rape, where a single L. biglobosa lesion on a leaf induced resistance throughout the leaf and other parts of the plant (Liu et al. 2006; Thomas et al. 2009). Induction of systemic resistance has also been demonstrated in other host-pathogen interactions following single leaf inoculations (Kuć 1982). Here, we show that mycovirus infection of $L$. biglobosa used to induce systemic resistance to L. maculans in oilseed rape significantly enhances resistance as 
compared with an isogenic virus-free isolate of L. biglobosa, and that previous investigations of this phenomena (Liu et al. 2006; Mahuku et al. 1996; Thomas et. al. 2009) most likely used virus-infected isolates of L. biglobosa to induce resistance. This was followed by next-generation sequencing (NGS) and transcriptome analysis to better understand how treatment with the virus-free versus a virus-infected isolate affects plant gene expression. These results illustrate the potential for deliberate inoculation of plants with hypervirulent L. biglobosa in crops on a larger scale to decrease the severity of Phoma stem canker later in the growing season.

\section{RESULTS AND DISCUSSION}

Isolation and identification of Leptosphaeria spp. from B. napus, screening for mycovirus double-stranded RNAs, and their cloning, elimination, and transfection.

Of the 73 field isolates of Leptosphaeria spp. studied in this investigation, PCR amplification of internal transcribed spacer (ITS) sequences revealed that 57 of them, including all 20 new field isolates from Cambridge, were L. maculans and the other 16 isolates were L. biglobosa (Supplementary Table S1). Normally, cultures of $L$. maculans have no pigment and are white while those of $L$. biglobosa produce a yellow pigment with numerous conidia on potato dextrose agar (PDA) (Fitt et al. 2006a). However the four Chinese isolates of L. biglobosa, belonging to the 'brassicae' subclade, were unusually pigmented white. Following small-scale isolation of double-stranded RNA (dsRNA) from all 73 isolates, it was discovered that none of the L. maculans contained any dsRNAs whereas 11 of 16 of the L. biglobosa isolates contained at least three or four dsRNA elements 4 to $4.9 \mathrm{kbp}$ in size. A representative GelRed-stained agarose gel of the dsRNA elements found in 8 of 11 representative L. biglobosa isolates is shown in Supplementary Fig. S1A. Following the production of a random cDNA library from these dsRNAs, clones were generated for sequencing which revealed that the virus was a novel member of the family Quadriviridae (Lin et al. 2012). Further cloning and genome walking with sequence-specific primers facilitated obtaining the complete sequence of the virus from the Chinese W10 isolate of L. biglobosa. The virus, named Leptosphaeria biglobosa quadrivirus-1 (LbQV1 ), is a member of the family Quadriviridae, and the complete molecular analysis of the LbQV-1 genome is the subject of a separate publication (Shah et al. 2019). Using the diagnostic reverse-transcription (RT)-PCR amplification procedure developed from the knowledge of the genomic sequence of LbQV-1, it was possible to confirm the presence of LbQV-1 in several isolates of L. biglobosa following the small-scale isolation of virus-specific dsRNAs (Supplementary Fig. S1B).

As shown in several other investigations with mycoviruses, it was possible to eliminate infection by LbQV-1 dsRNAs using cycloheximide (Kotta-Loizou and Coutts 2017a). However, when virus-infected $L$. biglobosa isolates were grown on solid media amended with cycloheximide it was found that concentrations of the drug $>0.5 \mathrm{mM}$ were toxic for the fungus and killed it, whereas concentrations $<0.1 \mathrm{mM}$ did not eliminate virus infection. Subsequently, a combinational approach of growing the fungus on V8 agar amended with $0.1 \mathrm{mM}$ cycloheximide and isolating single conidia on fresh agar without the drug was used in attempts to eliminate LbMV-1 infection from L. biglobosa C-Rox 12.8.1, KExc 12.10.21, and the four Chinese isolates. Between three and seven fungal colonies from each isolate were then assessed for the presence of the virus using both dsRNA isolation and RT-PCR amplification and only one of five colonies of the Chinese W10 L. biglobosa isolate was found to be virus free (Supplementary Fig. S1C). Purified LbQV-1 was then reintroduced into the virusfree W10-VF-1 using a transfection process whose success was verified by RT-PCR amplification (Supplementary Fig. S1D). The virus-transfected W10-VT-1, virus-free W10-VF-1, and virus-infected W10 isogenic lines obtained with this procedure were then used for further investigation.

Assessment of the effects of virus infection of $L$. biglobosa on colony morphology, mycelial growth rate, and biomass.

A comparison of the colony morphologies of the isogenic L. biglobosa $\mathrm{W} 10$ and $\mathrm{W} 10-\mathrm{VF}-1$ lines revealed significant phenotypes associated with virus infection in the former as compared with the latter following 26 days of culture on V8 agar plates. Virus-infected cultures of the L. biglobosa W10 isolate conidia formed a circular, distinct pattern of black pigmentation, which was more evident when the plates were observed from the back (Supplementary Fig. S2A). In contrast, the virus-free W10-VF-1 isolate was pigmented with no distinct pattern of sporulation and an undulate colony margin. These observations suggest that LbQV-1 infection significantly alters fungal pigmentation and growth.

A quantitative comparison of the mycelial growth rate of virus-infected $\mathrm{W} 10$, virus-free $\mathrm{W} 10-\mathrm{VF}-1$, and virus-transfected W10-VT-1 isogenic lines was made by measuring the radial expansion of fungal colonies on solid PDA over a 34day time course in at least three experiments. These results revealed that W10 and W10-VT-1, which harbor LbQV-1, grew significantly faster than W10-VF-1 over the time course $(P<$ 0.0001) (Supplementary Fig. S3A and B) and that these differences were visible throughout the culture period (Supplementary Fig. S2B). Similar comparisons of the effects of virus infection of $L$. biglobosa on fungal growth were made by investigating the biomass of W10, W10-VF-1, and W10-VT-1 produced in potato dextrose (PD) broth cultures. Here, significant increases in biomass for W10 and W10-VT-1 as compared with W10-VF-1 were found in at least three separate experiments $(P<0.0001)$ (Supplementary Fig. S3B). Taken together, these results emphatically demonstrate that infection of $L$. biglobosa with LbQV-1 results in increased growth.

\section{Effects of virus infection on the virulence of $L$. biglobosa on B. napus plants.}

The virulence of isogenic, virus-infected (W10) and virus-free (W10-VF-1) L. biglobosa lines was investigated following inoculation of B. napus 'Excel' cotyledons. Virulence was assessed regularly up to 15 days postinoculation (dpi) by inspecting cotyledons for the appearance of Phoma leaf spot lesions and noting increases in their area with time (Fig. 1A). Lesions appeared 4 dpi with W10 and 1 day later for W10-VF-1 but a comparison of the average lesion areas revealed that their sizes were not statistically significantly different from one another. However, from 5 to $8 \mathrm{dpi}$, the average lesion area increased slowly in cotyledons inoculated with W10-VF-1, whereas it increased rapidly in cotyledons inoculated with $\mathrm{W} 10$ and was significantly different (Fig. 1B; Table 1). At $8 \mathrm{dpi}$, the lesion area was greater on cotyledons inoculated with W10 as compared with W10-VF-1 and this trend was maintained 12 and $15 \mathrm{dpi}$, where statistically significant differences in the size of the lesion areas were found $(P<0.0001)$ (Fig. 1B; Table 1). These observations strongly suggest that the LbQV-1-infected L. biglobosa isolate W10 is hypervirulent on oilseed rape plants as compared with the corresponding virus-free, isogenic line $\mathrm{W} 10-\mathrm{VF}-1$ and confirm the hypervirulent effect of virus infection on growth of L. biglobosa found in synthetic media. The molecular mechanisms of this phenomena are, however, unknown.

Hypervirulence-associated traits of increased levels of conidiation and pigmentation may be related to the alteration of two signaling pathways: the cAMP-dependent and $\mathrm{Ca}^{2+}$. dependent pathways (Hillman et al. 2018). The upregulation of 
cAMP-dependent protein kinase and downregulation of $\mathrm{Ca}^{2+}$ dependent protein kinase are commonly observed in the 6-kbp dsRNA-induced hypervirulence in Nectria radicicola (Ahn and Lee 2001). Mild hypervirulence conferred by a four-segmented dsRNA virus of the human pathogen Aspergillus fumigatus (Kanhayuwa et al. 2015) and by a multisegmented virus complex of the entomopathogenic fungus Beauveria bassiana (Kotta-Loizou and Coutts 2017a) have been documented. More recently, a betachrysovirus was reported to cause hypervirulence to the plant-pathogenic fungus Alternaria alternata (Okada et al. 2018), while another betachrysovirus was found to convert the pathogenicity of its host, Magnaporthe oryzae, from avirulence to virulence and vice versa, depending on the rice variety (Aihara et al. 2018).

\section{NGS and data analysis of Brassica napus plants infected with L. biglobosa.}

To investigate the molecular mechanisms of virus-induced hypervirulence in planta, leaf tissue of Brassica napus inoculated with isogenic, virus-infected (W10) and virus-free (W10-VF-1)
L. biglobosa lines was collected and assessed using RNA sequencing. Alignment of the reads to the B. napus genome revealed that $>90 \%$ of the reads were derived from the plant, as observed in previous work for samples collected 7 dpi (Lowe et al. 2014). The relative percentage of reads derived from leaf tissue is similar in all samples (Supplementary Fig. S4A), whereas it is evident that B. napus inoculated with virus-infected (W10) L. biglobosa contains the highest relative percentage of fungal reads (Supplementary Fig. S4B), in agreement with the lesion measurements. Finally, RT quantitative PCR (RT-qPCR) amplification was used to confirm the presence of the virus exclusively in the plants inoculated with W10 (Supplementary Fig. S4C).

Following alignment of the reads to the B. napus transcriptome, quantification and normalization based on fragments per kilobase of gene per million mapped reads (FPKM), transcript expression was designated as negative $($ FPKM $<1)$, low $(1 \leq$ FPKM $<5)$, medium $(5 \leq$ FPKM $<25)$, or high (FPKM $\geq 25)$ (Fig. $2 \mathrm{~A})$, as described previously (Becker et al. 2017). It is evident that the major global effect of fungal infection involves gene expression upregulation (Fig. 2A and C). Differential expression analysis
A $\quad$ W10
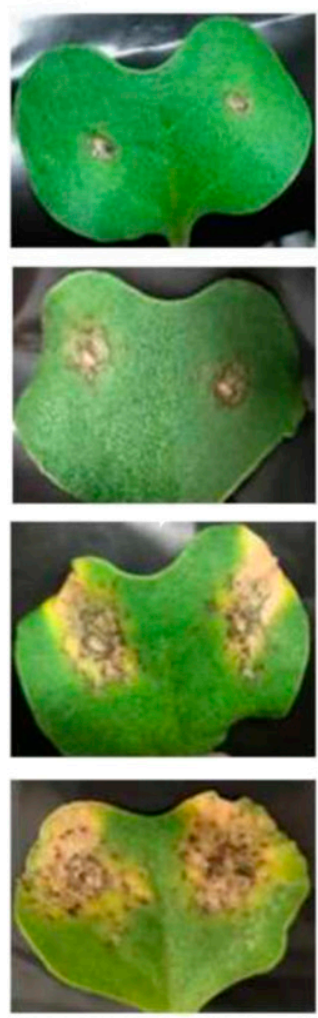

W10-VF-1
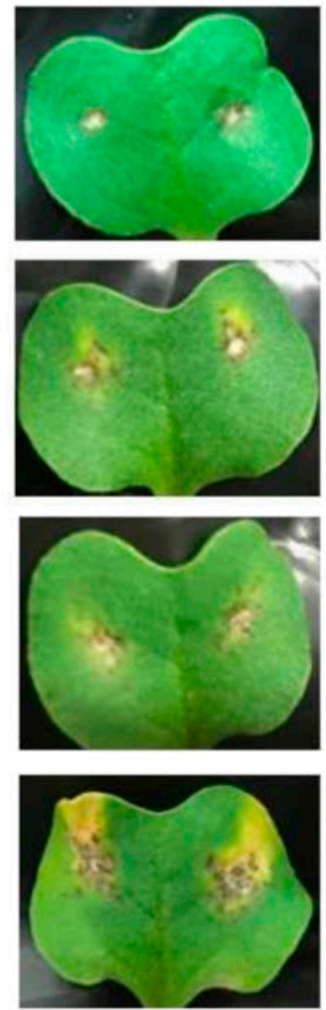

B

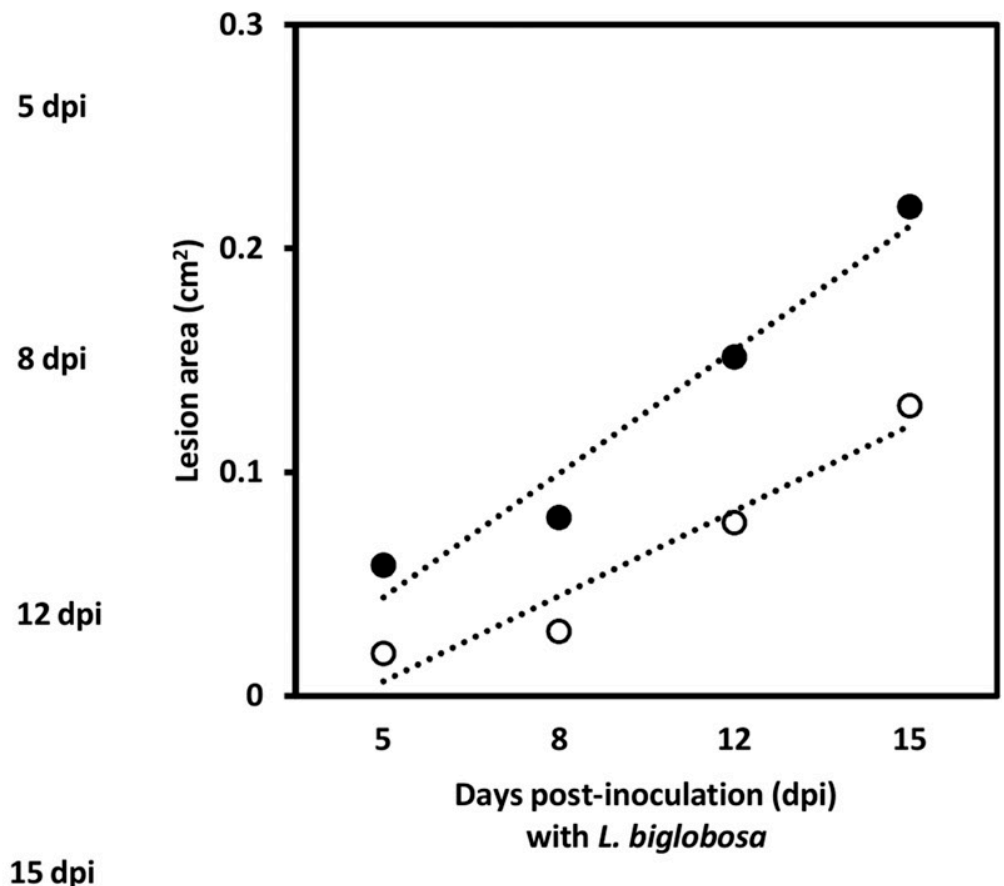

15 dpi

W10 O W10-VF-1

Fig. 1. A, Time course of development of Phoma leaf spots on Brassica napus 'Excel' cotyledons following inoculation with virus-infected W10 and virus-free W10-VF-1 isogenic lines of Leptosphaeria biglobosa at 5, 8, 12, and 15 days postinoculation (dpi). B, Mean lesion area for up to 40 lesions for each isolate $(P$ value $<0.001$; two-way analysis of variance), together with linear regression of the data. Parameters were estimated from the linear regression equation $y=a+b t$, where $y$ is average lesion area, $t$ is days postinoculation, and $a$ and $b$ are the intercept and slope parameters of the lines, respectively. For virus-infected W10 and virus-free W10-VF-1, the equations are $y=0.016 x-0.036$ and $y=0.011 x-0.049$, respectively.

Table 1. Lesion area for Leptosphaeria biglobosa virus-infected W10 and virus-free W10-VF-1 following inoculation of oilseed rape cotyledons, together with $P$ values following analysis of variance

\begin{tabular}{|c|c|c|c|c|}
\hline \multirow[b]{2}{*}{ Isolate } & \multicolumn{4}{|c|}{ Lesion area $\left(\mathrm{cm}^{2}\right)(\text { mean } \pm S D)^{a}$} \\
\hline & 5 dpi & 8 dpi & 12 dpi & 15 dpi \\
\hline W10 & $0.058 \pm 0.023$ & $0.079 \pm 0.033$ & $0.151 \pm 0.076$ & $0.218 \pm 0.132$ \\
\hline W10-VF-1 & $0.018 \pm 0.011$ & $0.028 \pm 0.012$ & $0.077 \pm 0.071$ & $0.129 \pm 0.078$ \\
\hline$P$ value (W10-VF-1 vs. W10) & NS & $<0.01$ & $<0.0001$ & $<0.0001$ \\
\hline
\end{tabular}

${ }^{\mathrm{a}} \mathrm{SD}=$ standard deviation, dpi = days postinoculation, and NS = not significant. 
revealed 4,705 (1,883 downregulated and 2,822 upregulated) and 1,942 (364 downregulated and 1,578 upregulated) transcripts affected in leaf tissue inoculated with W10-VF-1 and W10, respectively, when compared with untreated plant tissue (NGS SI).
In both cases, chloroplastic transcripts represent one-third to half of the downregulated dataset and $6 \%$ of the upregulated dataset (Fig. 2D), consistent with the leaf discoloration observed following infection.
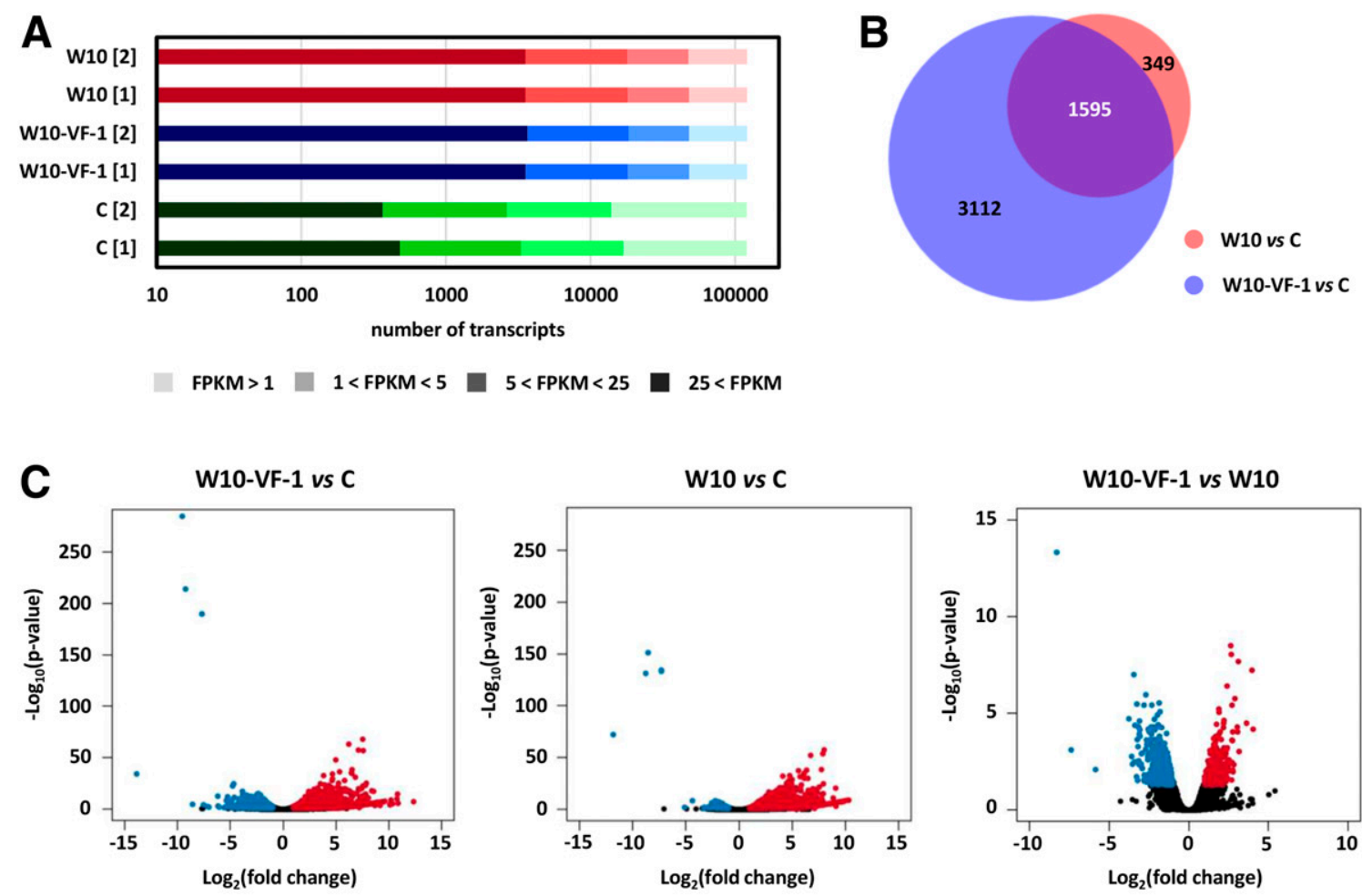

D

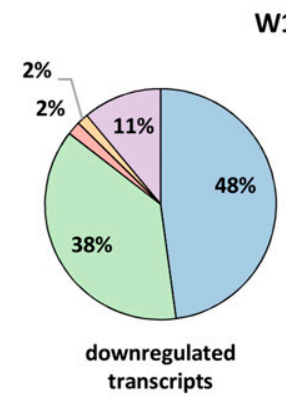

W10-VF-1 vs C

W10 vs C
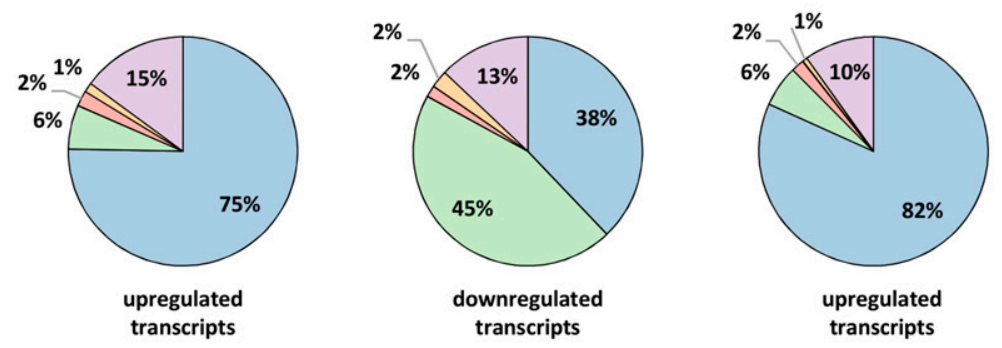

W10-VF-1 vs W10

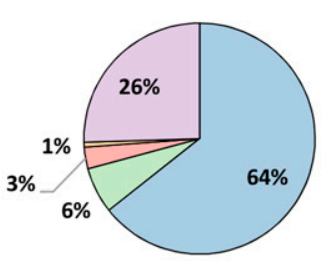

downregulated

transcripts

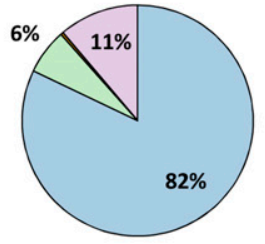

upregulated

transcripts

Fig. 2. A, Number of Brassica napus transcripts not expressed (fragments per kilobase of gene per million mapped reads $[$ FPKM] $<1$ ), expressed at low levels $(1<$ FPKM $<5)$, expressed at medium levels $(5<$ FPKM $<25)$, and expressed at high levels $(25<$ FPKM $)$ in each next-generation sequencing sample. B, Areaproportioned Venn diagram of transcripts differentially expressed in W10-treated versus untreated plants and W10-VF-1-treated versus untreated plants; "C" indicates the untreated control plants inoculated with water. C, Volcano plots following differential expression analysis; black dots signify $B$. napus transcripts whose expression is not significantly altered; red and blue dots signify differentially expressed ( $>2$-fold, $P$ value $<0.05)$ transcripts. D, Percentage of distinct species of transcripts down- and upregulated in B. napus inoculated with Leptosphaeria biglobosa W10 or W10-VF-1; pairwise comparisons for differential expression analysis include W10-treated versus untreated plants, W10-VF-1-treated versus untreated plants, and W10-VF-1-treated versus W10-treated plants. 
Five transcripts were significantly ( $>100$-fold) downregulated in B. napus infected by L. biglobosa compared with the control (Fig. 2C): three TIC 214-like proteins (XM_022701461, XM_022701462, and XR_002658990), part of the translocon on the inner chloroplast membrane responsible for preprotein transfer (Kikuchi et al. 2013); a noncoding RNA (XR_001285859), designated as uncharacterized but with significant identity $(>90 \%$; BLAST E-value 0.0) to the 5S ribosomal RNA (rRNA) gene region of other Brassica spp.; and another uncharacterized noncoding RNA

\section{Defensins}

defensin-like protein 1 (LOC106451698) defensin-like protein 3 (LOC106421680) defensin-like protein 1 (LOC106417637) defensin-like protein 1 (LOC106439300) defensin-like protein 2 (LOC106396331) defensin-like protein 197 (LOC106423952) defensin-like protein 1 (LOC106394443) defensin-like protein 3 (LOC106390979) defensin-like protein 197 (LOC106391835) defensin-like protein 1 (LOC106396110) defensin-like protein 32 (LOC111210322) X2 defensin-like protein 32 (LOC111210322) X1 defensin-like protein 4 (LOC106421519) defensin-like protein 195 (LOC106394617) defensin-like protein 195 (LOC106454543) defensin-like protein 195 (LOC106359651) defensin-like protein 195 (LOC106394639) defensin-like protein 3 (LOC106433348)

\section{W10-VF-1 vs C W10 vs C \\ B. napus + L. biglobosa}

endochitinase At2g43590-like (LOC106429247) endochitinase At2g43590-like (LOC106391825) endochitinase At2g43590 (LOC106385345) chitinase 10 (LOC106397849)

basic endochitinase C-like (LOC106374118) endochitinase $\mathrm{CH} 25$-like (LOC106398178) endochitinase $\mathrm{CH} 25$-like (LOC106353089) endochitinase CHI (LOC106388327) chitinase domain-containing protein 1-like (LOC106388754) chitinase 10 (LOC106430129) chitinase domain-containing protein 1-like (LOC106388758) endochitinase A-like (LOC106367652) chitinase domain-containing protein 1-like (LOC106438630) endochitinase At2g43620-like (LOC106388337) endochitinase At2g43620-like (LOC106388337)
endochitinase At2g43620-like (LOC111214050) endochitinase EP3 (LOC106412099)

chitinase-like protein 1 (LOC106365690) X1 chitinase-like protein 1 (LOC106365690) X2 chitinase-like protein 1 (LOC106371162) X1 chitinase-like protein 1 (LOC106371162) X2 chitinase-like protein 1 (LOC106371162) X2
chitinase-like protein 1 (LOC106401093) chitinase-like protein 1 (LOC106358812) basic endochitinase CHB4-like (LOC106389800) basic endochitinase CHB4-like (LOC106386585) endochitinase EP3-like (LOC106415445)

endochitinase At2g43590-like (LOC111205097) endochitinase EP3-like (LOC106412146) endochitinase EP3-like (LOC106415442) endochitinase CHI-like (LOC106396326)

basic endochitinase CHB4-like (LOC106360896) endochitinase $\mathrm{CHI}$-like (LOC106358854) endochitinase $\mathrm{CH} 25$-like (LOC106353093) basic endochitinase CHB4 (LOC106388328) basic endochitinase CHB4-like (LOC111197713) endochitinase At2g43580-like (LOC111215094) X1 endochitinase $\mathrm{CHI}-$ like (LOC106390154) endochitinase At2g43590-like (LOC106402913) endochitinase At2g43590-like (LOC106402912) endochitinase At2g43580-like (LOC111215094) X2 endochitinase $\mathrm{CH} 25$-like (LOC106452384 endochitinase $\mathrm{CH} 25$ (LOC106435387)
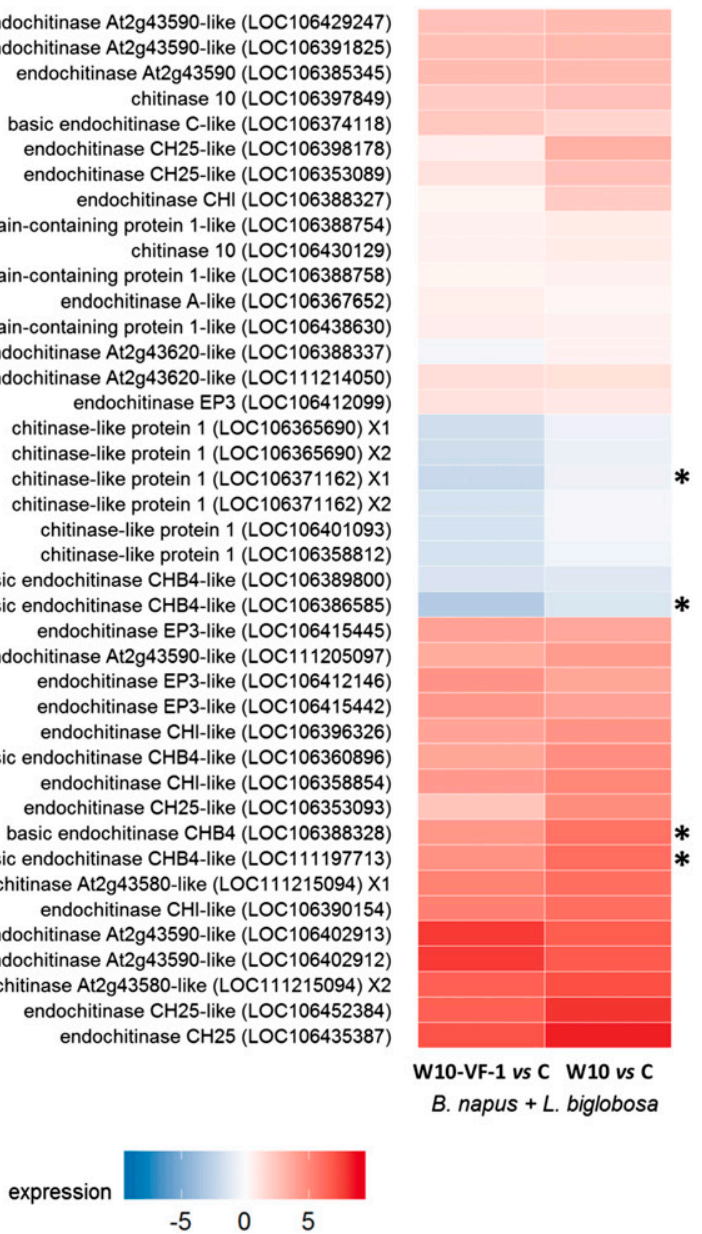

\section{Chitinases}

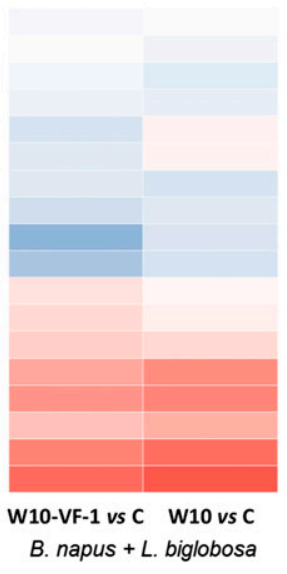

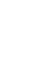

(XR_002658566), possibly associated with chloroplasts. Similarly, the three most upregulated transcripts include the pathogenesisrelated (PR) protein PR-4B (XM_013880327 and XM_013894987) and the PR protein 5-like (XM_013817307). Overall, a large number of transcripts associated with the $B$. napus defense response are differentially regulated, including PR proteins, defensins, chitinases, WRKY transcription factors, and hevein-like proteins (Fig. 3).

The B. napus genome has the potential to encode 52 PR-1 and PR-1-like proteins from distinct genetic loci. The proteins

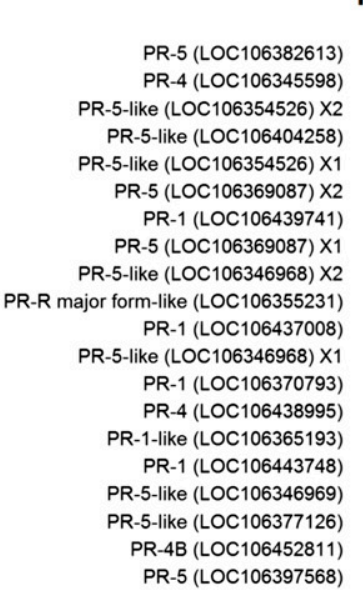

Pathogenesis-related proteins

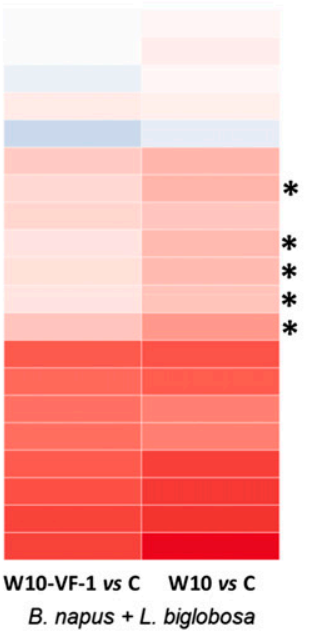

WRKY transcription factors

probable WRKY-75 (LOC106370642) WRKY-55-like (LOC106345444) X3 probable WRKY-28 (LOC106407432) WRKY-55-like (LOC106345444) X1 WRKY-55-like (LOC106345444) X2 probable WRKY-47 (LOC106426595) probable WRKY-48 (LOC111200856) probable WRKY-47 (LOC106430080) WRKY-6 (LOC106417496) WRKY-6-like (LOC111212223) probable WRKY-65 (LOC106360038) probable WRKY-31 (LOC106375978) X2 probable WRKY-15 (LOC106390291) probable WRKY-31 (LOC106437649) 06437649) WRKY-6 (LOC106401560) probable WRKY-45 (LOC111215820) WRKY-6-like (LOC111205252) probable WRKY-75 (LOC106431908) probable WRKY-8 (LOC106367627) probable WRKY-75 (LOC106379107) WRKY-6-like (LOC106442752) probable WRKY-65 (LOC106364696) probable WRKY-46 (LOC106394550) X2 probable WRKY-46 (LOC106449056) X1 probable WRKY-46 (LOC106394550) X1 probable WRKY-46 (LOC106394550) X3 WRKY-18-like (LOC106372529) WRKY-18-like (LOC106375397) probable WRKY-33 (LOC106405881) probable WRKY-33 (LOC106447673) probable WRKY-33 (LOC106447673)
probable WRKY-53 (LOC106443726) probable WRKY-70 (LOC106445333)

\section{W10-VF-1 vs C W10 vs C}

B. napus + L. biglobosa

\section{Hevein-like proteins}

hevein-like preproprotein (LOC106452809) hevein-like preproprotein (LOC106438996) hevein-like preproprotein (LOC106452810)

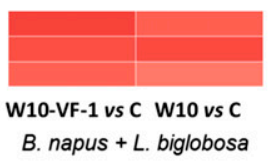

Fig. 3. Heat maps of normalized differential expression of transcripts involved in Brassica napus defense response after inoculation with Leptosphaeria biglobosa W10 and W10-VF-1, following hierarchical clustering. Asterisks indicate transcripts differentially upregulated in B. napus inoculated with W10-VF-1 compared with W10. 
encoded by these loci all belong to the CAP superfamily (cysteine-rich secretory proteins, antigen 5, and PR-1 proteins; PF00188) (El-Gebali et al. 2019) and are closely related to each other (Supplementary Fig. S5). As illustrated by NGS, only 5 transcripts were present in the leaves in sufficient quantity to allow for further studies and another 21 were above the limits of detection. The PR-1 proteins from loci LOC106437008 and LOC106439741 have 95\% similarity, while the PR-1 proteins from loci LOC106365193, LOC106370793, and LOC106443748 have $97 \%$ similarity. These transcripts are significantly upregulated following infection of B. napus with L. biglobosa, as shown by NGS and confirmed by RT-qPCR (Supplementary Fig. S6A and B). PR-1 proteins are upregulated during infection and high expression levels have been shown to result in increased plant resistance to fungal pathogens, although the exact mechanism is still unknown (Ali et al. 2018; Breen et al. 2017). Additionally, members of the WRKY family of transcription factors have also been implicated in the plant immune response (Chen et al. 2019). The B. napus genome has 429 distinct genetic loci predicted to encode WRKY transcription factors, and 32 of these were found to be differentially regulated following inoculation with L. biglobosa. The expression levels of two WRKY transcripts, WRKY-6-like (LOC111205252) and probable WRKY-75 (LOC106431908), were assessed by RTqPCR, confirming the significant upregulation shown by NGS (Supplementary Fig. S6C and D).

There are notable quantitative and qualitative differences in the number of B. napus transcripts affected by W10 and W10-VF-1 compared with the control. More specifically, the number of $B$. napus transcripts which are differentially expressed in a statistically significant manner following exposure to $\mathrm{W} 10$ are fewer than half of those affected as compared with W10-VF-1. These do not represent a mere subset of a larger dataset; transcripts specific for either W10 or W10VF-1 are noted in addition to the common ones (Fig. 2B). The proteins encoded by the differentially regulated $B$. napus transcripts were found to be involved in a range of Kyoto Encyclopedia of Genes and Genomes (KEGG) pathways, primarily metabolic (Supplementary Table S2). Transcripts differentially regulated exclusively by $\mathrm{W} 10-\mathrm{VF}-1$, were significantly overrepresented $(P$ value $<0.05)$ in carbohydrate and amino acid metabolic pathways, together with those leading to biosynthesis of various plant secondary metabolites, including the signaling molecules phenylpropanoids and antimicrobial metabolites such as alkaloids and glucosinolates (Matsuura and Fett-Neto 2015; Vig et al. 2009). Signal transduction, as represented by the plant mitogen-activated protein kinase signaling pathway, the phosphatidylinositol signaling system, and plant hormones, is also differentially affected by W10-VF-1.

Direct comparison of the transcriptome of B. napus inoculated with W10-VF-1 versus W10 revealed 703 (435 downregulated and 268 upregulated) differentially expressed transcripts. Confirming the previous analyses, these transcripts are involved in carbohydrate and amino acid metabolism but no more than $6 \%$ are associated with chloroplasts (Fig. 2D). Some transcripts associated with the $B$. napus defense response are also differentially affected (Supplementary Fig. S5). Gene ontology (GO) analysis also supported the effects on metabolism and enzymatic activities while linking differentially expressed transcripts with cell membrane localization and stress responses (Supplementary Fig. S7). The latter include abscisic acid, an isoprenoid plant hormone known to repress defenses against fungi (Jiang et al. 2010; Schmidt et al. 2008; Spence et al. 2015), which would partly explain the observed hypervirulent effect. Differential expression analysis of the L. biglobosa transcriptome did not provide any further insights into the plant-fungus interactions because no differentially expressed transcripts were identified, potentially due to the low number of reads derived from L. biglobosa.

\section{Pretreatment of $B$. napus plants with $L$. biglobosa and challenge inoculation with $L$. maculans.}

When the first true leaves of B. napus 'Excel' plants were preinoculated with virus-infected W10 L. biglobosa conidia, the sizes of the lesions elicited following challenge inoculation with L. maculans conidia on second true leaves increased slowly whereas, in plants preinoculated with virus-free, W10VF-1 L. biglobosa conidia or water, the average lesion area on challenged leaves increased rapidly up to 13 dpi (Fig. 4A). Statistically significant decreased lesion sizes on challengeinoculated leaves 15 dpi $(P<0.0001)$ were noted for plants preinoculated with virus-infected $L$. biglobosa as compared with those plants preinoculated with virus-free L. biglobosa or water, both of which were similar to each other and not significantly different (Fig. 4B; Table 2). The rate of increase in leaf lesion area elicited by L. maculans challenge inoculation on the second true leaves was significantly slower in plants preinoculated with virus-infected L. biglobosa as compared with virus-free L. biglobosa or control water preinoculated plants (Fig. 4B; Table 2). Interestingly, transcripts encoding PR proteins, mostly PR-1-like and PR-5-like, are expressed in significantly higher levels in B. napus after inoculation with virus-infected L. biglobosa as compared with virus-free L. biglobosa (Fig. 3). PR-1 and PR-5 proteins have been associated with SAR in plants (Ali et al. 2018) and their subtle differential regulation may partially explain the LbQV-1-mediated SAR effect.

Several studies have illustrated that severity of Phoma stem canker disease of $B$. napus and B. juncea were reduced when plants were artificially inoculated with nonaggressive L. biglobosa 'canadensis' (Mahuku et al. 1996; Thomas et al. 2009) or L. biglobosa 'brassicae' (Liu et al. 2006) (present study) prior to or simultaneous with later inoculation with aggressive L. maculans isolates. Because similar results were reported in these studies, the L. biglobosa inoculum sources used to induce systemic resistance whether conidiospores (Thomas et al. 2009) (present study) or ascospores (Liu et al. 2006) are used would not appear to be important. However, we show here, for the first time, that virus infection of the inducing L. biglobosa strain is important in the induction of resistance because there was no significant induction against challenge inoculation with $L$. maculans when virus-free isolates were used for preinoculation of lower leaves. The frequency of LbQV-1 infection of field isolates of L. biglobosa investigated in this study (namely, 11 of 16) is remarkably high and, because of this, we suspect that it is likely that other investigations that used L. biglobosa to induce systemic resistance to L. maculans in oilseed rape used LbQV-1-infected isolates, although we cannot prove this. In the future, we plan to assess the effect that deliberate prior inoculation of oilseed rape with LbQV-1infected L. biglobosa has on the later stages of blackleg disease, particularly collaring and canker, following L. maculans infection in field situations.

\section{MATERIALS AND METHODS}

\section{Isolation and identification of Leptosphaeria spp. from B. napus; screening for mycovirus dsRNAs; and cloning, elimination, and transfection of dsRNAs.}

In total, 73 Leptosphaeria isolates obtained from oilseed rape (B. napus) leaves with Phoma leaf spot symptoms or from stems with stem canker symptoms were investigated, including 20 isolates from a single field site near Cambridge, U.K. Individual Leptosphaeria isolates were obtained using the method 
of West et al. (2002). Leaves with Phoma leaf spots were washed with water and dried, and individual spots were excised, placed in Petri dishes on Whatman number 1 filter paper, sprayed with distilled water, and incubated at $20^{\circ} \mathrm{C}$ for 3 to 5 days under a regime of $12 \mathrm{~h}$ of light and $12 \mathrm{~h}$ of darkness to induce conidia production. Stems with cirrhi from individual mature conidia were collected using a fine needle, mixed with a drop of sterilized water to make a conidial suspension, transferred onto V8 agar plates, and incubated for 5 days at $20^{\circ} \mathrm{C}$ in darkness to produce confluent cultures (Zhang et al. 2014). To identify individual Leptosphaeria spp. (whether L. maculans or L. biglobosa) and L. biglobosa subclades, isolates were subcultured on PDA plates and identified on the basis of morphological phenotype and by PCR amplification of the rRNA region incorporating the ITS regions and the 5.8S rRNA gene (LbigF: 5'-ATC AGG GGATTG GTG TCA GCA GTT GA-3' and LbigR: 5'-GCA AAATGT GCT GCG CTC CAG G-3') (Liu et al. 2006; Liu et al. 2014).

All Leptosphaeria isolates were screened for the presence of mycovirus dsRNA elements using a small-scale dsRNA extraction protocol (Coenen et al. 1997). Extracts were treated with DNase I and SI nuclease to remove host-contaminating nucleic acids and agarose gel electrophoresis was done to assess the presence, size, and purity of the dsRNAs following staining with ethidium bromide (Kotta-Loizou and Coutts 2017a). cDNA clones were produced using a random priming procedure for dsRNAs (Froussard 1992), cloned, and sequenced. The sequences were analyzed to identify homology with previously characterized sequences in the database using the BLAST programs (Altschul et al. 1997). Subsequently, the complete genomic sequence of a novel virus named LbQV-1 was obtained. Knowledge of the LbQV-1 genomic sequence facilitated development of a diagnostic RT-PCR amplification procedure. This procedure generated a 317-bp amplicon and used total fungal RNA extracts (RNeasy; Qiagen) and oligonucleotide primers based on the LbQV-1 largest dsRNA (5'CAC CAG CAT ACT GCA GGG AA-3' and 5'-GCC TTG TCG TGA GTA CAC CA-3'), which is a genetic component of all quadriviruses (Kotta-Loizou and Coutts 2017b).

In order to determine the effects of mycovirus infection on fungal growth and virulence, it was essential to generate virusfree and virus-infected isogenic lines which could be compared. To eliminate virus infection, one isolate (W10) was grown on V8 agar containing 0.01 to $50 \mathrm{mM}$ cycloheximide for 20 days
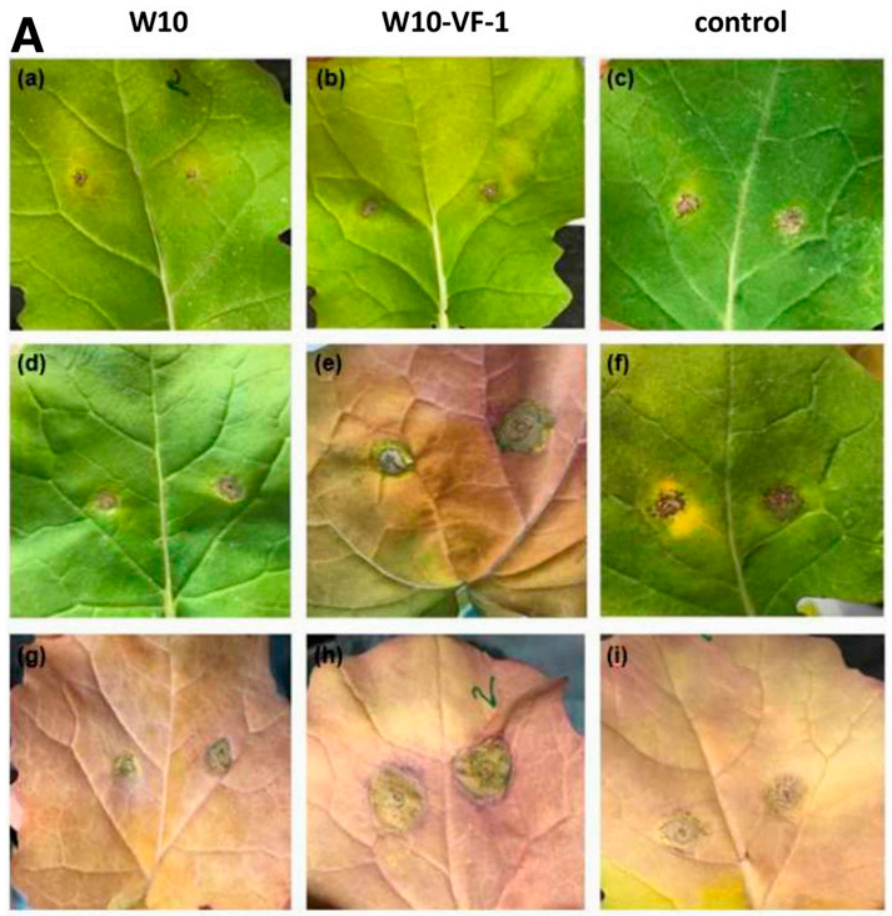

B

$13 \mathrm{dpi}$

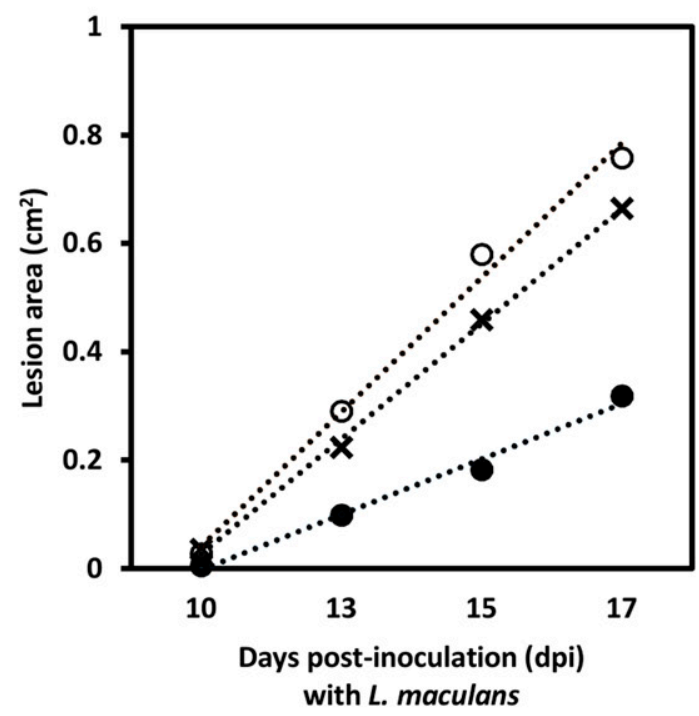

- W10 OW10-VF-1 x control

Fig. 4. A, Effect of preinoculation of oilseed rape first leaves with virus-infected W10 (a, d, and g) or virus-free W10-VF-1 (b, e, and h) Leptosphaeria biglobosa on development of Phoma leaf spot lesions on second true leaves following challenge inoculation with $L$. maculans conidia at 13 (a, b, and c), 15 (d, $\mathrm{e}$, and f), and 17 (g, h, and i) days postinoculation (dpi). Control first true leaves were inoculated with sterile water (c, f, and i). B, Mean lesion area for up to 18 lesions on oilseed rape second true leaves challenge inoculated with $L$. maculans conidia following preinoculation of the first true leaves with water (control), virus-infected W10, or virus-free W10-VF-1 L. biglobosa ( $P$ value $<0.001$; two-way analysis of variance), together with linear regression of the data. For virusinfected W10, virus-free W10-VF-1, and water (control), the equations are $y=0.044 x-0.45, y=1.060 x-0.107$, and $y=0.091 x-0.905$, respectively.

Table 2. Lesion area on oilseed rape second true leaves challenge inoculated with Leptosphaeria maculans conidia following preinoculation of the first true leaves with water (control), virus-infected W10, or virus-free W10-VF-1 L. biglobosa, together with $P$ values following analysis of variance

\begin{tabular}{|c|c|c|c|c|}
\hline \multirow[b]{2}{*}{ Isolate } & \multicolumn{4}{|c|}{ Lesion area $\left(\mathrm{cm}^{2}\right)(\text { mean } \pm \mathrm{SD})^{\mathrm{a}}$} \\
\hline & 10 dpi & 13 dpi & 15 dpi & 17 dpi \\
\hline W10 & $0.005 \pm 0.009$ & $0.098 \pm 0.062$ & $0.182 \pm 0.076$ & $0.318 \pm 0.117$ \\
\hline W10-VF-1 & $0.026 \pm 0.033$ & $0.289 \pm 0.131$ & $0.579 \pm 0.200$ & $0.757 \pm 0.184$ \\
\hline Control & $0.035 \pm 0.038$ & $0.223 \pm 0.042$ & $0.459 \pm 0.194$ & $0.664 \pm 0.359$ \\
\hline$P$ value (W10-VF-1 vs. W10) & NS & $<0.01$ & $<0.0001$ & $<0.0001$ \\
\hline
\end{tabular}

${ }^{\mathrm{a}} \mathrm{SD}=$ standard deviation, dpi = days postinoculation, and NS = not significant. 
at $20^{\circ} \mathrm{C}$. Single conidia were then recovered on V8 medium without cycloheximide and were assessed for the presence of LbQV-1 by isolating dsRNA using the small-scale procedure and RT-PCR amplification of a fragment of the RNA-dependent RNA polymerase gene. LbQV-1 was then reintroduced in the virus-free isogenic line in order to complete Koch's postulates. Transfection was performed using a procedure similar to that described previously (Kanhayuwa et al. 2015; Kotta-Loizou and Coutts 2017a), following generation of L. biglobosa protoplasts from fresh mycelia grown for 32 to $36 \mathrm{~h}$ and digested with $3.2 \%$ (wt/vol) Trichoderma harzianum lysing enzyme (Glucanex, L-1412; Sigma) and $0.1 \mathrm{~g}$ of cellulase (Onozuka, 201089; Yakult Honsha Co.).

\section{Preparation of $L$. biglobosa conidial suspensions} for assessment of fungal growth and virulence.

L. biglobosa cultures were grown on V8 agar plates under a regime of $12 \mathrm{~h}$ of light and $12 \mathrm{~h}$ of darkness to induce sporulation. Sporulating cultures were covered with sterile distilled water and the agar surface was scraped with a Lazy-L spreader to release conidia into the water. Conidia were collected after filtering the suspension through Miracloth and their numbers were counted using a hemocytometer prior to adjusting their concentration by dilution with water for various inoculations.

\section{Assessment of the effects of virus infection of $\boldsymbol{L}$. biglobosa on colony morphology, mycelial growth rate, and biomass. \\ The phenotype and colony morphology of the virus-infected L. biglobosa $\mathrm{W} 10$ isolate and the virus-free, W10-VF-1 iso- genic line, both grown on V8 agar, were compared over a 26- day incubation period at $20^{\circ} \mathrm{C}$. Quantitative assessment of growth was done by measuring the radial expansion of colonies over a 34-day incubation period at $20^{\circ} \mathrm{C}$ following central in- oculation of PDA plates with conidia $\left(n=10^{6}\right)$ of each of the isogenic lines. Time periods were determined in such a way that fungal growth had commenced before the first measurements were taken and the last measurements were taken when the mycelium had reached the Petri dish edge. To determine bio- mass production by the isogenic lines, equal numbers of con- idia $\left(n=2 \times 10^{6}\right)$ of each of the isolates were inoculated into 250-ml flasks containing $150 \mathrm{ml}$ of PD broth and incubated at $20^{\circ} \mathrm{C}$ on a rotary shaker $(130 \mathrm{rpm})$ for 13 days. The mycelium from individual cultures was then harvested by centrifugation at $15,000 \times g$ for $20 \mathrm{~min}$ and the resultant pellets were lyophilized for dry weight determination. All experiments were done in triplicate.}

\section{Effects of virus infection on the virulence of $L$. biglobosa on B. napus plants.}

Seed of Excel winter oilseed rape (B. napus) were germinated and grown at $20^{\circ} \mathrm{C}$ in Petri dishes on Whatman filter paper after spraying with distilled water. For cotyledon inoculations, germinated seed were sown in seed trays containing equal amounts of Miracle Gro and John Innes number 3 compost placed in larger plastic trays $(49 \mathrm{~cm}$ long and $40 \mathrm{~cm}$ wide) filled with water and maintained at 20 to $24^{\circ} \mathrm{C}$ under natural light (supplemented with fluorescent light; $300 \mu \mathrm{E} \mathrm{m}^{-2} \mathrm{~s}^{-1}$ ). Plants were selected for inoculation 14 days later and, after removal of any developing true leaves, were supported with sticks. At the edge of one of the cotyledon lobes, a small hole was made for identification purposes. Each lobe was wounded at the center with a sharp pin prior to inoculation with $10 \mu \mathrm{l}$ of a conidial suspension $\left(10^{7}\right.$ conidia $\left.\mathrm{ml}^{-1}\right)$ of either the L. biglobosa W10 or W10-VF-1 isolates. All plants were then transferred into a controlled-environment cabinet $\left(20^{\circ} \mathrm{C}\right.$ day, $20^{\circ} \mathrm{C}$ night, regime of $12 \mathrm{~h}$ of light and $12 \mathrm{~h}$ of darkness at a light intensity of $210 \mu \mathrm{E} \mathrm{m}^{-2} \mathrm{~s}^{-1}, 80$ to $85 \%$ relative humidity
$[\mathrm{RH}])$ and sprayed daily to maintain moisture and high humidity. Disease severity was assessed by measuring lesion area (length by width) at the two inoculation sites for each cotyledon up to $15 \mathrm{dpi}$. The incubation period was estimated as the time taken after inoculation for the first lesions to develop. Up to 32 lesion areas for each isolate (mean \pm standard deviation) were measured in three separate experiments. Significant differences in lesion area were calculated using two-way analysis of variance implemented by GraphPad PRISM 6 tests and the data were subjected to linear regression where the parameters were estimated from the linear regression equation $y=a+b t$, where $y$ is average lesion area, $t$ is days postinoculation, and $a$ and $b$ are the intercept and slope parameters of the lines, respectively.

\section{Pretreatment of $B$. napus 'Excel' plants with $L$. biglobosa and challenge inoculation with $L$. maculans.}

For first-true-leaf plant inoculations, seed were germinated and, after 7 days, individual seedlings were transplanted into 9-cm-diameter pots containing compost. Prior to inoculation, 4-week-old plants were arranged in a randomized, incomplete block design with two replicates, with 9 plants/treatment for each replicate (totaling 27 plants), and transferred to a controlled environment room and incubated for 2 days at $15^{\circ} \mathrm{C}$ with 60 to $80 \% \mathrm{RH}$ and a regime of $16 \mathrm{~h}$ of light and $8 \mathrm{~h}$ of darkness at a light intensity of 300 to $350 \mu \mathrm{E} \mathrm{m}^{-2} \mathrm{~s}^{-1}$. All pots were then placed in plastic trays ( $49 \mathrm{~cm}$ long and $40 \mathrm{~cm}$ wide) which were kept filled with water $(1 \mathrm{~cm}$ in depth) throughout the experiments. For pretreatment with L. biglobosa (virus-infected or virus-free), small regions ( $7 \mathrm{~mm}$ in diameter) of the first true leaves were abraded gently with a cylindrical eraser so that they could hold droplets of the conidial suspensions (Huang et al. 2001). Then, each abraded area was wounded with a sterile needle and a $12-\mu$ d droplet of conidia $\left(10^{7}\right.$ conidia $\left.\mathrm{ml}^{-1}\right)$ was pipetted onto the wounded area. Inoculated plants were then covered with a lid and black polyethylene sheets and moved into plant growth chambers. Control pretreatments used sterile distilled water inoculation of abraded leaves. Plants were then incubated in the dark under conditions of high humidity ( 80 to $85 \% \mathrm{RH}$ ) for $24 \mathrm{~h}$ and the lids removed $24 \mathrm{~h}$ later (i.e., $48 \mathrm{hpi}$ ). At $96 \mathrm{~h}$ after pretreatment, the second true leaves of plants were challenge inoculated with $10 \mu \mathrm{l}$ of a conidial suspension (L. maculans isolate H Rox 12.2.1 (avrLm4 and avrLm7 at $10^{7}$ conidia ml ${ }^{-1}$ ) (Mitrousia et al. 2018). All challenge-inoculated plants were maintained under the same conditions for $48 \mathrm{~h}$ and subsequently incubated uncovered in the controlledenvironment cabinet. The times taken for second true leaves to develop lesions (brown to gray in color) were recorded for each plant challenge inoculated with $L$. maculans. Thereafter, lengths and widths of lesions on challenge-inoculated leaves were measured at different time points up until $17 \mathrm{dpi}$ and statistical differences between the pretreatments were calculated and analyzed by regression.

\section{NGS and data analysis of $B$. napus plants infected with $L$. biglobosa.}

$B$. napus whole leaves inoculated with a conidial suspension of either the L. biglobosa W10 or the W10-VF-1 isolates and collected 7 dpi were assessed using NGS. RNA extraction, rRNA depletion, library construction, and Illumina sequencing were performed by Genewiz. The overall quality of the sequencing data were assessed using FASTQC, and adapters and low-quality bases were clipped and trimmed, respectively, from the reads using Trimmomatic (Bolger et al. 2014). The reads were mapped to the $B$. napus genome and transcriptome (assembly GCA_000686985.2) using HISAT2 (Kim et al. 2015) and Bowtie2 (Langmead and Salzberg 2012), respectively, and gene expression was quantified by Salmon (Patro et al. 2017). 
The reads were also mapped to the L. biglobosa G12-14 genome (Dutreux et al. 2018) and gene expression was quantified by HTSeq (Anders et al. 2015). Differential gene expression analysis was done with DESeq2 (Love et al. 2014) and a transcript was considered differentially expressed in a statistically significant manner if it was up- or downregulated at least twofold with an adjusted $P$ value $<0.05$. The hypergeometric function phyper was implemented in R/Bioconductor, GO analysis was done using BLAST2GO (Götz et al. 2008), and the resulting GO terms were reduced and visualized by REVIGO (Supek et al. 2011) using the Arabidopsis thaliana database for calculating GO term sizes. KEGG pathways were identified using KAAS (Moriya et al. 2007) and custom scripts were written in Perl. Phylogenetic analysis of all annotated B. napus PR-1 protein sequences was performed using MEGA 6 (Tamura et al. 2013), following alignment using MUSCLE, and maximum-likelihood phylogenetic trees were constructed using the JTT+I+G substitution model. The presence of LbQV-1 in the leaves and the levels of the selected plant transcripts were confirmed by RT followed by RT-qPCR assays, performed in the OneStepPlus Real-Time qPCR System (Applied Biosystems) using the Power SYBR Green PCR Master Mix (Applied Biosystems) and the relative standard curve quantitation method (Kotta-Loizou and Coutts 2017a). Targetspecific primer pairs were designed using Primer-BLAST (Ye et al. 2012) for LbQV-1 (5'-CAC CAG CAT ACT GCA GGG AA- $3^{\prime}$ and $5^{\prime}$-GCC TTG TCG TGA GTA CAC CA- $3^{\prime}$ ), the two PR-1 protein groups (5'-AAA CGC TCA CAA CCA AGC AC$3^{\prime}$ and $5^{\prime}$-AGA AAA GTC GGC GCT ACT CC-3'; 5'-GGT GGG TCC TTT AAG GTG GG-3' and $5^{\prime}$-AGT CAA ATT GCT CCT TCA CCC-3'), the WRKY-6-like transcription factor (5' GTG ACA ACG TAG AGG ACG CT-3' and 5'-TCG TCG TCG GTC ACT GAT TG-3'), and the putative WRKY-75 transcription factor $\left(5^{\prime}\right.$-AGG AGA AGA AGA GGC CTC GAA-3' and 5' -AGT AGC TCC TAG GGA ACG TGT-3'). The $B$. napus TIP41 sequence served as endogenous control because it has been reported as a stably expressed reference gene (Chen et al. 2010) and was amplified using target-specific primer pairs (5'-AGA GTC ATG CCA AGT TCA TGG TT-3' and 5' -CCT CAT AAG CAC ACC ATC AAC TCT AA-3').

\section{ACKNOWLEDGMENTS}

We thank Y. Huang for supplying U.K. Leptosphaeria isolates; G. Li for supplying Chinese L. biglobosa isolates; G. Mitrousia for advice on their identification using PCR amplification procedures; A. Qi for advice on statistical analysis; and C. Filippou, D. Ogbeni, and L. Bruno for technical assistance.

\section{LITERATURE CITED}

Ahn, I. P., and Lee, Y. H. 2001. A viral double-stranded RNA up regulates the fungal virulence of Nectria radicicola. Mol. Plant-Microbe Interact. 14:496-507.

Aihara, M., Urayama, S. I., Le, M. T., Katoh, Y., Higashiura, T., Fukuhara, T., Arie, T., Teraoka, T., Komatsu, K., and Moriyama, H. 2018. Infection by Magnaporthe oryzae chrysovirus 1 strain A triggers reduced virulence and pathogenic race conversion of it host fungus, Magnaporthe oryzae. J. Gen. Plant Pathol. 84:92-103.

Ali, S., Ganai, B. A., Kamili, A. N., Bhat, A. A., Mir, Z. A., Bhat, J. A., Tyagi, A., Islam, S. T., Mushtaq, M., Yadav, P., Rawat, S., and Grover, A. 2018. Pathogenesis-related proteins and peptides as promising tools for engineering plants with multiple stress tolerance. Microbiol. Res. 212213:29-37.

Altschul, S. F., Madden, T. L., Schäffer, A. A., Zhang, J., Zhang, Z., Miller, W., and Lipman, D. J. 1997. Gapped BLAST and PSI-BLAST: A new generation of protein database search programs. Nucleic Acids Res. 25: 3389-3402.

Anders, S., Pyl, P. T., and Huber, W. 2015. HTSeq-A Python framework to work with high-throughput sequencing data. Bioinformatics 31:166-169.
Becker, M. G., Zhang, X., Walker, P. L., Wan, J. C., Millar, J. L., Khan, D., Granger, M. J., Cavers, J. D., Chan, A. C., Fernando, D. W. G., and Belmonte, M. F. 2017. Transcriptome analysis of the Brassica napusLeptosphaeria maculans pathosystem identifies receptor, signaling and structural genes underlying plant resistance. Plant J. 90:573-586.

Bolger, A. M., Lohse, M., and Usadel, B. 2014. Trimmomatic: A flexible trimmer for Illumina sequence data. Bioinformatics 30:2114-2120.

Breen, S., Williams, S. J., Outram, M., Kobe, B., and Solomon, P. S. 2017. Emerging insights into the functions of pathogenesis-related protein 1. Trends Plant Sci. 22:871-879.

Chen, X., Li, C., Wang, H., and Guo, Z. 2019. WRKY transcription factors: Evolution, binding, and action. Phytopathol. Res. 1:13.

Chen, X., Truksa, M., Shah, S., and Weselake, R. J. 2010. A survey of quantitative real-time polymerase chain reaction internal reference genes for expression studies in Brassica napus. Anal. Biochem. 405:138-140.

Coenen, A., Kevei, F., and Hoekstra, R. F. 1997. Factors affecting the spread of double-stranded RNA viruses in Aspergillus nidulans. Genet. Res. 69: $1-10$.

Dutreux, F., Da Silva, C., d'Agata, L., Couloux, A., Gay, E. J., Istace, B., Lapalu, N., Lemainque, A., Linglin, J., Noel, B., Wincker, P., Cruaud, C., Rouxel, T., Balesdent, M. H., and Aury, J. M. 2018. De novo assembly and annotation of three Leptosphaeria genomes using Oxford Nanopore MinION sequencing. Sci. Data 5:180235.

El-Gebali, S., Mistry, J., Bateman, A., Eddy, S. R., Luciani, A., Potter, S. C., Qureshi, M., Richardson, L. J., Salazar, G. A., Smart, A., Sonnhammer E. L. L., Hirsh, L., Paladin, L., Piovesan, D., Tosatto, S. C. E., and Finn, R. D. 2019. The Pfam protein families database in 2019. Nucleic Acids Res. 47:D427-D432.

Fitt, B. D. L., Brun, H., Barbetti, M. J., and Rimmer, S. R. 2006a. Worldwide importance of Phoma stem canker (Leptosphaeria maculans and L. biglobosa) on oilseed rape (Brassica napus). Eur. J. Plant Pathol. 114: 3-15.

Fitt, B. D. L., Huang, Y.-J., van den Bosch, F., and West, J. S. 2006b. Coexistence of related pathogen species on arable crops in space and time. Annu. Rev. Phytopathol. 44:163-182.

Froussard, P. 1992. A random-PCR method (rPCR) to construct whole cDNA library from low amounts of RNA. Nucleic Acids Res. 20:2900

Götz, S., García-Gómez, J. M., Terol, J., Williams, T. D., Nagaraj, S. H., Nueda, M. J., Robles, M., Talón, M., Dopazo, J., and Conesa, A. 2008. High-throughput functional annotation and data mining with the Blast2GO suite. Nucleic Acids Res. 36:3420-3435.

Hillman, B. I., Annisa, A., and Suzuki, N. 2018. Viruses of plant-Interacting fungi. Adv. Virus Res. 100:99-116.

Huang, Y. J., Toscano-Underwood, C., Fitt, B. D. L., Todd, A. D., West, J. S., Koopmann, B., and Balesdent, M. H. 2001. Effects of temperature on germination and hyphal growth from ascospores of A-group and Bgroup Leptosphaeria maculans (Phoma stem canker of oilseed rape). Ann. Appl. Biol. 139:193-207.

Jiang, C. J., Shimono, M., Sugano, S., Kojima, M., Yazawa, K., Yoshida, R., Inoue, H., Hayashi, N., Sakakibara, H., and Takatsuji, H. 2010. Abscisic acid interacts antagonistically with salicylic acid signaling pathway in rice-Magnaporthe grisea interaction. Mol. Plant-Microbe Interact. 23: 791-798.

Kanhayuwa, L., Kotta-Loizou, I., Özkan, S., Gunning, A. P., and Coutts, R. H. A. 2015. A novel mycovirus from Aspergillus fumigatus contains four unique dsRNAs as its genome and is infectious as dsRNA. Proc. Natl. Acad. Sci. U.S.A. 112:9100-9105.

Kikuchi, S., Bédard, J., Hirano, M., Hirabayashi, Y., Oishi, M., Imai, M., Takase, M., Ide, T., and Nakai, M. 2013. Uncovering the protein translocon at the chloroplast inner envelope membrane. Science 339: 571-574.

Kim, D., Langmead, B., and Salzberg, S. L. 2015. HISAT: A fast spliced aligner with low memory requirements. Nat. Methods 12:357-360.

Kotta-Loizou, I., and Coutts, R. H. A. 2017a. Studies on the virome of the entomopathogenic fungus Beauveria bassiana reveal novel dsRNA elements and mild hypervirulence. PLoS Pathog. 13:e1006183.

Kotta-Loizou, I., and Coutts, R. H. A. 2017b. Mycoviruses in Aspergilli: A comprehensive review. Front. Microbiol. 8:1699.

Kuć, J. 1982. Induced immunity to plant disease. BioScience 32:854-860.

Langmead, B., and Salzberg, S. L. 2012. Fast gapped-read alignment with Bowtie 2. Nat. Methods 9:357-359.

Lin, Y.-H., Chiba, S., Tani, A., Kondo, H., Sasaki, A., Kanematsu, S., and Suzuki, N. 2012. A novel quadripartite dsRNA virus isolated from a phytopathogenic filamentous fungus, Rosellinia necatrix. Virology 426: 42-50.

Liu, S. Y., Liu, Z., Fitt, B. D. L., Evans, N., Foster, S. J., Huang, Y. J., Latunde-Dada, A. O., and Lucas, J. A. 2006. Resistance of Leptosphaeria maculans (Phoma stem canker) in Brassica napus (oilseed rape) 
induced by L. biglobosa and chemical defence activators in field and controlled environments. Plant Pathol. 55:401-412.

Liu, Z., Latunde-Dada, A. O., Avice, M., Hall, A. M., and Fitt, B. D. L. 2014. Phoma stem canker disease on oilseed rape (Brassica napus) in China is caused by Leptosphaeria biglobosa 'brassicae'. Eur. J. Plant Pathol. 140:841-857.

Love, M. I., Huber, W., and Anders, S. 2014. Moderated estimation of fold change and dispersion for RNA-seq data with DESeq2. Genome Biol. 15:550.

Lowe, R. G. T., Cassin, A., Grandaubert, J., Clark, B. L., Van de Wouw, A. P., Rouxel, T., and Howlett, B. J. 2014. Genomes and transcriptomes of partners in plant-fungal-interactions between canola (Brassica napus) and two Leptosphaeria species. PLoS One 9:e103098.

Mahuku, G. S., Hall, R., and Goodwin, P. H. 1996. Co-infection and induction of systemic acquired resistance by weakly and highly virulent isolates of Leptosphaeria maculans in oilseed rape. Physiol. Mol. Plant Pathol. 49: 61-72.

Matsuura, H. N., and Fett-Neto, A. G. 2015. Plant alkaloids; main features, toxicity and mechanism of action. Pages 1-15 in: Plant Toxins. Toxinology. P. Gopalakrishnakone, C. Carlini, and R. Ligabue-Braun, eds. Springer, Dordrecht, The Netherlands.

Mitrousia, G. K., Huang, Y. J., Qi, A., Sidique, S. N. M., and Fitt, B. D. L. 2018. Effectiveness of Rlm7 resistance against Leptosphaeria maculans (Phoma stem canker) in UK winter oilseed rape cultivars. Plant Pathol. 67:1339-1353.

Moriya, Y., Itoh, M., Okuda, S., Yoshizawa, A. C., and Kanehisa, M. 2007. KAAS: An automatic genome annotation and pathway reconstruction server. Nucleic Acids Res. 35:W182-W185.

Okada, R., Ichinose, S., Takeshita, K., Urayama, S. I., Fukuhara, T., Komatsu, K., Arie, T., Ishihara, A., Egusa, M., Kodama, M., and Moriyama, H. 2018. Molecular characterization of a novel mycovirus in Alternaria alternata manifesting two-sided effects: Down-regulation of host growth and upregulation of host plant pathogenicity. Virology 519:23-32.

Patro, R., Duggal, G., Love, M. I., Irizarry, R. A., and Kingsford, C. 2017. Salmon provides fast and bias-aware quantification of transcript expression. Nat. Methods 14:417-419.

Pieterse, C. M., Zamioudis, C., Berendsen, R. L., Weller, D. M., Van Wees, S. C., and Bakker, P. A. 2014. Induced systemic resistance by beneficial microbes. Annu. Rev. Phytopathol. 52:347-375.
Schmidt, K., Pflugmacher, M., Klages, S., Mäser, A., Mock, A., and Stahl, D. J. 2008. Accumulation of the hormone abscisic acid (ABA) at the infection site of the fungus Cercospora beticola supports the role of ABA as a repressor of plant defence in sugar beet. Mol. Plant Pathol. 9: 661-673.

Shah, U. A., Kotta-Loizou, I., Fitt, B. D. L., and Coutts, R. H. A. 2019. Identification, molecular characterization, and biology of a novel quadrivirus infecting the phytopathogenic fungus Leptosphaeria biglobosa. Viruses 11:9.

Spence, C. A., Lakshmanan, V., Donofrio, N., and Bais, H. P. 2015. Crucial roles of abscisic acid biogenesis in virulence of rice blast fungus Magnaporthe oryzae. Front. Plant Sci. 6:1082.

Supek, F., Bošnjak, M., Škunca, N., and Šmuc, T. 2011. REVIGO summarizes and visualizes long lists of gene ontology terms. PLoS One 6:e21800.

Tamura, K., Stecher, G., Peterson, D., Filipski, A., and Kumar, S. 2013. MEGA6: Molecular Evolutionary Genetics Analysis version 6.0. Mol. Biol. Evol. 30:2725-2729.

Thomas, V. L., Norton, R. M., Marcroft, S. J., and Salisbury, P. A. 2009. A Leptosphaeria biglobosa 'canadensis' isolate induced resistance in Brassica juncea L. and Brassica napus L. against Leptosphaeria maculans (Desm.) Ces. et de Not. Proc. 16th Aust. Res. Assembly on Brassicas. Ballarat, Victoria, Australia.

Vig, A. P., Rampal, G., Thind, T. S., and Arora, S. 2009. Bio-protective effects of glucosinolates-A review. LWT-Food. Sci. Technol. 42: 1561-1572.

West, J. S., Fitt, B. D. L., Leech, P. K., Biddulph, J. E., Huang, Y. J., and Balesdent, M. H. 2002. Effects of timing of Leptosphaeria maculans ascospore release and fungicide regime on Phoma leaf spot and Phoma stem canker development on winter oilseed rape (Brassica napus) in southern England. Plant Pathol. 51:454-463.

Ye, J., Coulouris, G., Zaretskaya, I., Cutcutache, I., Rozen, S., and Madden, T. L. 2012. Primer-BLAST: A tool to design target-specific primers for polymerase chain reaction. BMC Bioinf. 13:134.

Zhang, X., White, R. P., Demir, E., Jedryczka, M., Lange, R. M., Islam, M., Li, Z. Q., Huang, Y. J., Hall, A. M., Zhou, G., Wang, Z., Cai, X., Skelsey, P., and Fitt, B. D. L. 2014. Leptosphaeria sp., Phoma stem canker and potential spread of L. maculans on oilseed rape crops in China. Plant Pathol. 63:598-612. 